\title{
COUNTEREXAMPLES ON THE RANK OF A MANIFOLD ${ }^{1}$
}

\author{
GLEN E. BREDON
}

\begin{abstract}
For any given integer $r$ a closed manifold is constructed which has a smooth free action of the $r$-torus, and hence has rank at least $r$, but for which -1 is not a multiple root of the Poincaré polynomial.
\end{abstract}

Let $M^{n}$ be an orientable, closed, differentiable $n$-manifold. The rank of $M$ is defined to be the maximum number of (everywhere) independent commuting vector fields on $M$. The Poincaré polynomial of $M$ is $P_{M}(t)=\sum b_{i} t^{i}$ where $b_{i}$ is the $i$ th Betti number of $M$. Thus rank $M \geqq 1$ iff -1 is a root of $P_{M}(t)$. It has been conjectured (see $\left[1\right.$, p. 669]) that if rank $M>1$ then -1 is a multiple root of $P_{M}(t)$. A weaker conjecture is that if the 2-torus $T$ acts freely and smoothly on $M$ (i.e., $M$ is the total space of a principal $T$-bundle) then -1 is a multiple root of $P_{M}(t)$.

This conjecture is so attractive that perhaps no serious attempt has been made to disprove it. At least that is the only explanation for the simplicity of the counterexamples which we shall now give.

If $A^{k} \subset M^{n}$ is a $k$-dimensional closed submanifold of $M$, then we denote by $d(M, A)$ the $n$-manifold obtained by removing an open tubular neighborhood of $A$ from $M$ and doubling the remainder along its boundary. Let us compute the Poincaré polynomial of $d(M, A)$. We shall assume that

$$
\tilde{H}_{*}(A) \rightarrow \widetilde{H}_{*}(M) \text { is trivial and } n \geqq 2 k+3
$$

partially for convenience of computation, but mostly since the conclusions would be false without some such assumptions.

If $M_{0}$ is the complement of an open tube about $A$, then $M_{0} \subset M$ induces an isomorphism in homology through the middle dimension, since $\operatorname{dim} A=k \leqq(n-3) / 2$. The boundary of $M_{0}$ is an $(n-k-1)$ sphere bundle over $A$ and $k<n-k-1$, by assumption, so that $H_{p}\left(\partial M_{0}\right) \approx H_{p}(A)$ for $p \leqq n-k-2$. Since $n-k-2 \geqq[n / 2]$, this holds through the middle dimension. The Mayer-Vietoris sequence

Received by the editors April 3, 1970.

AMS 1970 subject classifications. Primary 57D25, 57E15, 58A30.

Key words and phrases. Commuting vector fields, Poincaré polynomial, free toral action.

1 This research was supported in part by NSF grant GP-11468. 


$$
\begin{aligned}
\cdots \rightarrow H_{p}\left(\partial M_{0}\right) & \stackrel{i_{*}}{\rightarrow} H_{p}\left(M_{0}\right) \oplus H_{p}\left(M_{0}\right) \stackrel{j_{*}}{\rightarrow} H_{p}(d(M, A)) \\
& \stackrel{\partial_{*}}{\rightarrow} H_{p-1}\left(\partial M_{0}\right) \rightarrow \cdots
\end{aligned}
$$

has $i_{*}=0$ (for $p \neq 0$ ) at least through the middle dimension, by the assumption that $A$ is homologically trivial in $M$. Thus, through the middle dimension, $P_{d(M, A)}(t)$ coincides with $2 P_{M}(t)-1+t\left(P_{A}(t)-1\right)$. By Poincaré duality, we conclude that

$$
\begin{aligned}
P_{d(M, A)}(t) & =2 P_{M}(t)-1-t^{n}+t\left(P_{A}(t)-1\right)+t^{n-k-1}\left(P_{A}(t)-t^{k}\right) \\
& =2 P_{M}(t)+\left(t+t^{n-k-1}\right) P_{A}(t)-(1+t)\left(1+t^{n-1}\right) .
\end{aligned}
$$

Now suppose that $n$ is odd and that the $r$-torus $T^{r}(r \geqq 2)$ acts freely on $M$ with $A$ invariant. Also suppose that $(1+t)^{2}$ divides both $P_{M}(t)$ and $P_{A}(t)$. (The simplest example is $r=2, k=2, M=S^{3} \times S^{3}$ $\times S^{1}$ and $A=T^{2}$ an orbit of the obvious $T^{2}$ action on the first two factors of $S^{3} \times S^{3} \times S^{1}$.) Then $T^{r}$ also acts freely on $d(M, A)$, so that $\operatorname{rank}(d(M, A)) \geqq r$, but

$$
P_{d(M, A)}(t) \equiv-(1+t)\left(1+t^{n-1}\right) \not \equiv 0\left[\bmod (1+t)^{2}\right],
$$

(for $n$ odd), so that -1 is a simple root of $P_{d(M, A)}(t)$. Note that, for example, we can take $M=S^{m} \times S^{m} \times \cdots \times S^{m}$ (an odd number $\geqq r \geqq 2$ of copies, and $m$ odd) with $T^{r}=S^{1} \times \cdots \times S^{1}$ acting in the standard way on the first $r$ "coordinates," and $A$ can be taken to be $S^{p} \times \cdots \times S^{p} \times * \times \cdots \times *\left(r\right.$ copies of $S^{p}$ and $p$ odd $)$ for $m$ much larger than $p$. Then $d(M, A)$ is $p$-connected. Thus we have

THEOREM A. There are manifolds of arbitrarily high rank and connectivity whose Poincaré polynomials have -1 as a simple root.

For $n$ even, the Poincaré polynomial cannot have -1 as a simple root, since if $P_{M}(t)=(1+t) Q(t)$, then $Q(t)$ obeys formal Poincaré duality and has odd degree, whence $Q(-1)=0$. Note however that if $(1+t)^{3}$ divides both $P_{M}(t)$ and $P_{A}(t)$, then (for $n$ even)

$$
\begin{array}{r}
P_{d(M, A)}(t) \equiv-(1+t)\left(1+t^{n-1}\right)=-(1+t)^{2}\left(1-t+t^{2}-\cdots+t^{n-2}\right) \neq 0 \\
\quad\left[\bmod (1+t)^{3}\right] .
\end{array}
$$

Taking an even number of copies of $S^{m}$ in the above example, we then have

THEOREM B. There are even dimensional manifolds of arbitrarily high rank and connectivity whose Poincare polynomials have -1 as (only) a double root. 
These examples would seem to indicate that, despite intuition, the rank of a manifold and the multiplicity of -1 as a root of its Poincaré polynomial, have very little to do with each other.

\section{REFERENCES}

1. P. E. Thomas, Vector fields on manifolds, Bull. Amer. Math. Soc. 75 (1969), 643683.

Rutgers University, New Brunswick, New Jersey 08903 